\title{
Brief description of laser polarimetry microscopy methods of optically anisotropic biological layers
}

\author{
A.G. Ushenko, A.V. Dubolazov, V.A. Ushenko, Yu.A. Ushenko, M.Yu. Sakhnovskiy, \\ O.V. Olar, A.V. Motrich, P.O. Angelsky, M.P. Gorsky \\ Chernivtsi National University, 2, Kotsyubinsky str., 58012 Chernivtsi,Ukraine; e-mail: a.dubolazov@chnu.edu.ua
}

\begin{abstract}
The results of optical modeling biological tissues polycrystalline multilayer networks have been presented. Algorithms providing reconstruction of parameter distributions were determined, which enabled to describe linear and circular birefringence in these tissues. To separate manifestations of the respective mechanisms, we propose the method for space-frequency filtering. Criteria for differentiation of benign and malignant tissues of the women reproductive sphere have been found.
\end{abstract}

Keywords: polarization tomography, biological crystals, diagnostics

Manuscript received 11.07.16; revised version received 16.09.16; accepted for publication 16.11.16; published online 05.12.16.

1. Linear and circular birefringence in biological tissues

\subsection{Introduction}

Among the numerous areas of optical diagnostics aimed at the structure of biological tissues, widely used are techniques that use laser polarized radiation [1-3]. Particularly, laser polarimetry of microscopic images of polycrystalline protein networks was formed as a separate approach in the study of optically anisotropic components of various biological tissues [4-29].

The purpose of our research is development of the experimental method based on Fourier polarimetry and spatial-frequency selection of parameter distributions characteristic for linear and circular birefringence observed in the biopsy of the uterine wall tissue in order to differentiate benign (fibromyoma) and malignant (adenocarcinoma) conditions.

\subsection{Theory of the method}

The following model concepts have been formulated by us [14] as the basis to analyze the processes of amplitude-phase modulation of laser radiation by the polycrystalline network inherent to biological crystals with linear and circular birefringence:

- the uterine wall tissue consists of two optically anisotropic layers of the muscular (myometrium) and connective (endometrium) layers;

- phase anisotropy of these layers formed by optically active myosin and collagen proteins that form the fibrillar network with different conformational structure;

- the optically anisotropic myometrium network is formed by spatially ordered myosin fibrils. This spatial organization generates large-scale (the range of the transverse dimensions $l \sim 50 \ldots 300 \mu \mathrm{m}$ ) optically uniaxial birefringent linear structure. 
Polarization properties of biological crystals is characterized by the Jones matrix of the following form

$$
\left.\frac{7}{5} \| \begin{array}{ll}
\sin ^{2} \rho+\cos ^{2} \rho \exp \leftarrow i \delta ; & \sin \rho \cos \rho(-\exp \& i \delta \\
\sin \rho \cos \rho(-\exp \& i \delta ; & \cos ^{2} \rho+\sin ^{2} \rho \exp \leftarrow i \delta
\end{array}\right] \text {, }
$$

where $\rho$ is the direction of their axes, determines the direction of stacking fibrils, $\delta=\frac{2 \pi l}{\lambda} \Delta n$-value of the phase shift between orthogonal components of the amplitude, $\lambda$ - wavelength, $\Delta n$ - linear birefringence;

- the optically anisotropic component of the endometrium is formed by small-scale $(l \sim$ $5 . .25 \mu \mathrm{m})$ randomly oriented collagen fibrils with predominantly circular birefringence

$$
A \frac{7}{J}\left\|\begin{array}{cc}
\cos \theta & \sin \theta \\
-\sin \theta & \cos \theta
\end{array}\right\| \text {, }
$$

where $\theta=\frac{\pi l}{\lambda} \Delta n_{ \pm}$is the angle of the polarization plane; $\Delta n_{ \pm}-$indicator of the circular birefringence.

- the process of converting laser radiation by optically anisotropic fibrils is characterized by the following matrix equation

$$
E=Q F_{0} \text {. }
$$

From the medical viewpoint, "optic separation" of different mechanisms of anisotropy inherent to layers of uterine wall is a topical task. The fact is that at the initial, early stages the pathological changes are accompanied by a concentration increase of optically active proteins in polycrystalline network of myometrium and endometrium. Fibrillar structural changes - formation of the growth direction of tumour shoots and so on correspond to a more developed pathological condition.

With the purpose to select these conditions, we have applied the method of spatial-frequency filtration of the polarization inhomogeneous field $E_{x} \boldsymbol{\phi}, \delta, \theta_{-}^{-}$and $E_{y} \boldsymbol{\phi}, \delta, \theta^{-}$in the Fourier plane. The main idea of this approach is that the spatial-frequency structure of the Fourier form for laser image of the tissue of uterine wall is different in its large-scale (myosin) and small-scale (collagen) protein structures. Proceeding from this, using spatial-frequency filtration, it is mainly possible to isolate either low-frequency (with linear birefringence) or high-frequency (with circular birefringence) components, which by means of Fourier conversion can be transformed into respective "separated" laser images.

In our case, we consider the situation when the distance between projection lens, the layer of the object under study, and the plane of registration constitute focal distance $f$.

$U_{x} \varangle, \mu=\frac{1}{i \lambda f} \int_{-\infty}^{\infty} \int E_{x} \varangle, y \exp -i 2 \pi(v+y \mu \stackrel{-}{\underline{L}} d x d y,(6)$ $\delta$ and circular $\theta$ anisotropy of fibrillar network layers myometrium and endometrium can be found from the corresponding Jones matrix (relation (4) and (5)).

$$
\begin{aligned}
& \delta \boldsymbol{q}_{i k}=\arccos \left\{\frac{i \boldsymbol{E}_{y} E_{x}^{*}-E_{x} E_{y}^{*} \oslash}{\boldsymbol{E}_{y} E_{x}^{*}+E_{x} E_{y}^{*} \circledast}-\right.
\end{aligned}
$$

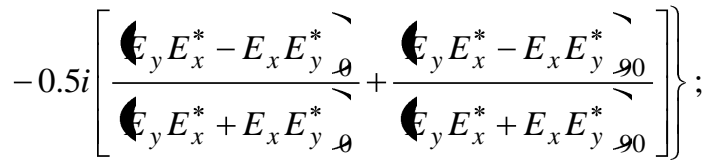

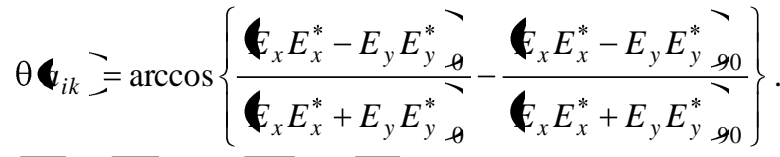

Here, we use the following notation:

* - complex conjugate;

- $\otimes-$ sensing the right circularly polarized wave $E_{0}=\left(\begin{array}{l}1 \\ i\end{array}\right)$

- 0 - sounding a linearly polarized wave $E_{0}=\left(\begin{array}{l}1 \\ 0\end{array}\right)$ with the azimuth $0^{\circ}$;

- 90 - sounding a linearly polarized wave $E_{0}=\left(\begin{array}{l}0 \\ 1\end{array}\right)$ with the azimuth $90^{\circ}$.

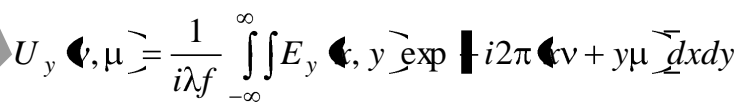

Here, $U_{x}, U_{y}$ are Fourier images of distributions $E_{x} \boldsymbol{\phi}, \delta, \theta_{-}^{-}$and $E_{y} \boldsymbol{\phi}, \delta, \theta^{-}$in the focal plane with the spatial frequencies $\nu=\frac{x}{\lambda f}$ and $\mu=\frac{y}{\lambda f}$.

If we place the vignetting transparent $R \wedge v, \Delta \mu_{-}$or opaque $R^{-1} \wedge \nu, \Delta \mu$ - diaphragm into the central part of Fourier plane, we can identify the corresponding areas of the spatial-frequency structure of the distributions amplitudes that are formed by predominant influences of linear $\hat{U} \boldsymbol{\phi}, \delta, \nu, \mu$, and circular $\dot{U} \boldsymbol{\theta}, \nu, \mu$ - birefringence

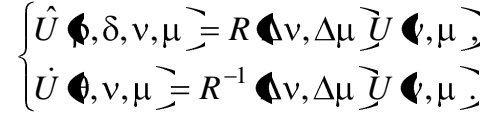

To renew the proper distributions of complex amplitudes $\left\{\begin{array}{l}\hat{E}_{x} \boldsymbol{\phi}, \delta, x, y ; \\ \dot{E}_{x} \boldsymbol{\theta}, x, y-\end{array}\right.$ and $\left\{\begin{array}{l}\hat{E}_{y} \boldsymbol{\phi}, \delta, x, y_{2} \\ \dot{E}_{y} \boldsymbol{\theta}, x, y\end{array}\right.$ in the image plane of this layer is possible by reverse Fourier transformation 


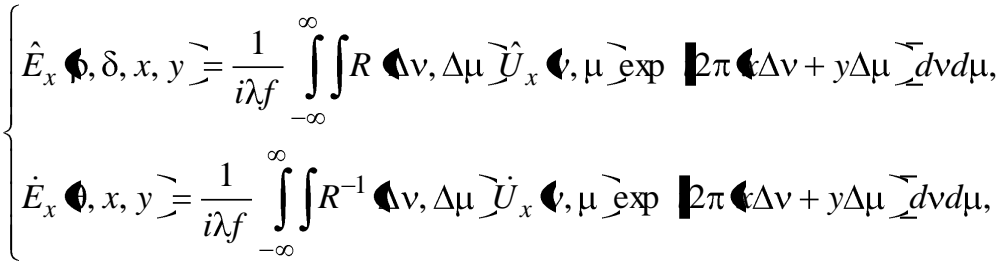

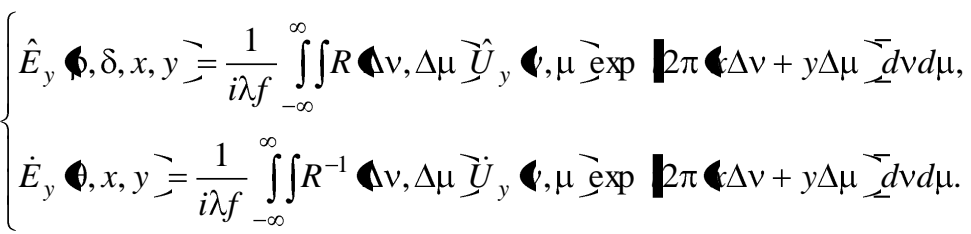

Within each pixel digital camera kits, the measured intensities are as $R \rightarrow I_{\circledast}^{\otimes} ; \quad I_{\oplus}^{\otimes} ; \quad I_{\circledast}^{0} ; \quad I_{\oplus}^{0} ; \quad I_{\circledast}^{90} ; \quad I_{\oplus}^{90}$ follows: $R^{-1} \rightarrow I_{0}^{0} ; \quad I_{90}^{0} ; \quad I_{0}^{90} ; \quad I_{90}^{90}$. The magnitude of phase shift $\delta \boldsymbol{R}_{-}^{-}$and rotation of plane polarization $\theta \mathbf{R}^{-1}$. were calculated in accord with the following equations.

$$
\begin{aligned}
& \delta \boldsymbol{R}_{-}^{-}=\arccos \left\{\frac{\mathbf{\otimes}_{\otimes}^{\otimes}-I_{\oplus}^{\otimes}}{\mathbf{⿴ 囗 十}_{\otimes}^{\otimes}+I_{\oplus}^{\otimes}},-\right.
\end{aligned}
$$

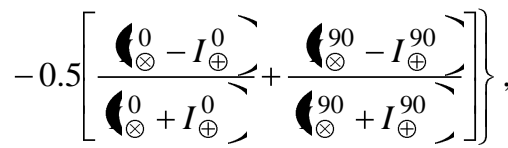$$
\left.\theta \boldsymbol{R}^{-1}=0.5 \arccos \left\{\frac{\mathbf{(}_{0}^{0}-I_{90}^{0}-}{\mathbf{l}_{0}^{0}+I_{90}^{0}-}-\frac{\mathbf{(}_{0}^{90}-I_{90}^{90}}{\mathbf{(}_{0}^{90}+I_{90}^{90}}\right\}\right\} .
$$

To estimate distributions $q=\left\{\begin{array}{ll}\delta\left\lfloor, n^{-}\right. \\ \theta\left(n, n^{\prime}\right.\end{array}\right.$, we calculated a set of statistical moments of the $1^{\text {st }}$ to $4^{\text {th }}$ orders $Z_{j=1 ; 2 ; 3 ; 4}$ using the following algorithms [2]

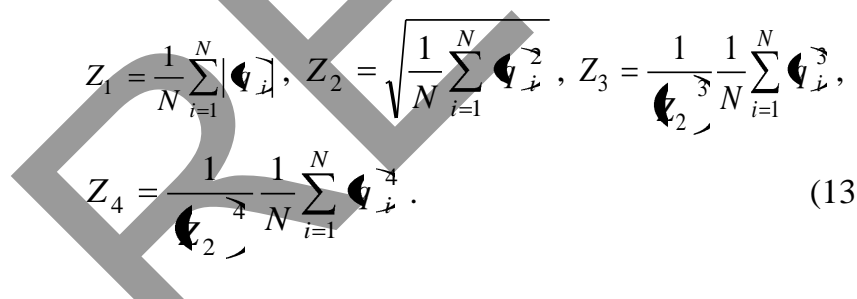

\section{Spatial-frequency polarimetry of optically anisotropic network in biological tissues}

As objects of investigation, we chose two groups of optically-thin (attenuation coefficient $\tau \approx 0.09 \ldots 0.095$ ) histological sections taken as biopsy of benign (26 samples) and malignant (26 samples) tumor of the uterus. The samples were prepared using a freezing microtome and the standard technique.

\subsection{Spatial-frequency Fourier polarimetry of linear birefringence in the fibrilar matrices of uterine tissue}

For the purpose of choosing optimal conditions for spatial-frequency filtration, we used the following range $\Delta r=2 \ldots 50$ pix of accessible dimensions for the vignetting diaphragm. As a criterion for choosing the size of aperture was an extreme change in the set of statistical moments of the $1^{\text {st }}$ to $4^{\text {th }}$ orders $Z_{j=1 ; 2 ; 3 ; 4}$ (Eq. (13)) that characterize the distribution $\delta \mu_{-}$. In our case, obtained was the following optimal size $\Delta r=$ 30 pix. This geometric size was chosen for comparative investigations of optical anisotropy in the fibrilar networks of uterine tissue with benign and malignant changes (Fig. 1).

Quantitatively, the differences between phase maps of the protein matrices in uterine tissue of both types are illustrated by the average values and standard deviations of the set of statistic $Z_{i=1 ; 2 ; 3 ; 4}$ parameters are summarized in Table 1. Separate figures give the specificity $S p=a(a+b)^{-1}(a, b$ are the numbers of correct and incorrect conclusions) of these parameters in differentiation of benign and malignant uterine tumors.

A comparative analysis of the data obtained using Fourier polarimetry of linear birefringence in the protein polycrystalline networks of histological sections of benign and malignant uterine tumors biopsy revealed sensitivity to differentiation of these states $\left(Z_{i=1 ; 2 ; 3 ; 4}\right)$ with the specificity level $S e \sim 63 \ldots 79 \%$ (printed in grey color in Table 1).

Table 1. Parameters of the statistic, correlative and selfsimilar structure of phase maps for linear birefringence in the polycrystalline uterine tissue networks.

\begin{tabular}{|c|c|c|c|}
\hline \multirow{2}{*}{ Parameters } & \multicolumn{2}{|c|}{$\delta(m \times n)$} & \multirow{2}{*}{$S p, \%$} \\
\cline { 2 - 3 } & Fibromyoma & Adenocarcinoma & \\
\hline$Z_{1}$ & $0.32 \pm 0.059$ & $0.48 \pm 0.11$ & 73 \\
\hline$Z_{2}$ & $0.13 \pm 0.022$ & $0.23 \pm 0.031$ & 65 \\
\hline$Z_{3}$ & $1.56 \pm 0.37$ & $0.82 \pm 0.14$ & 79 \\
\hline$Z_{4}$ & $0.85 \pm 0.21$ & $0.59 \pm 0.051$ & 83 \\
\hline
\end{tabular}




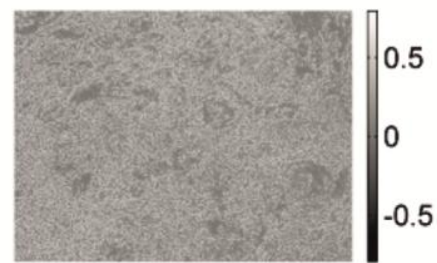

a)

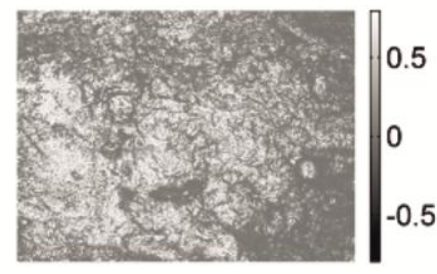

c)

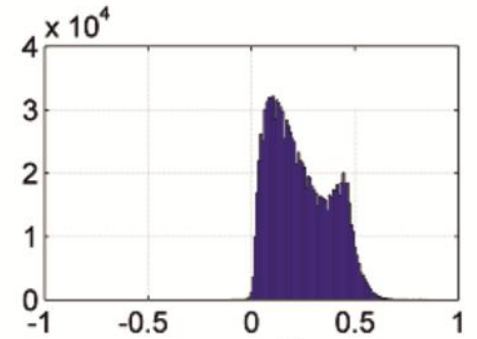

b)

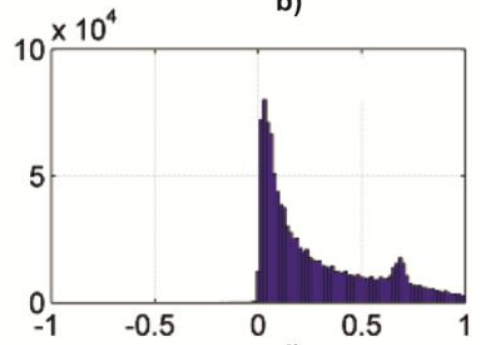

d)

Fig. 1. "Low-frequency" coordinate maps (a), (c) and histograms (b), (d) of the linear birefringence distribution for the histological section of benign uterine tumor (fibromyoma) biopsy ((a), (b)) and malignant uterine tumor (adenocarcinoma) biopsy $((\mathrm{c}),(\mathrm{d}))$.

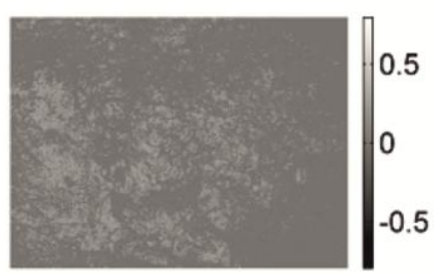

a)

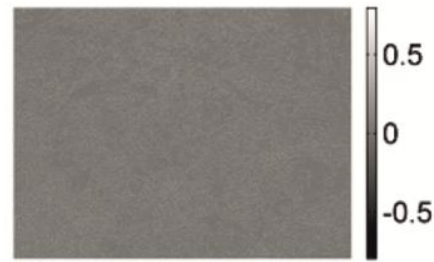

c)

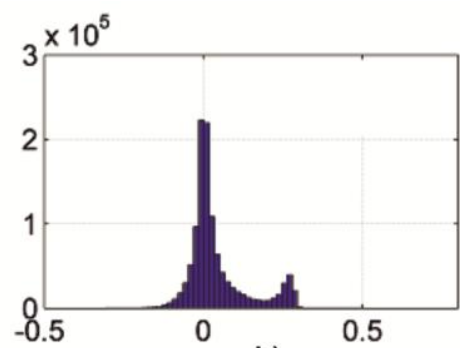

b)

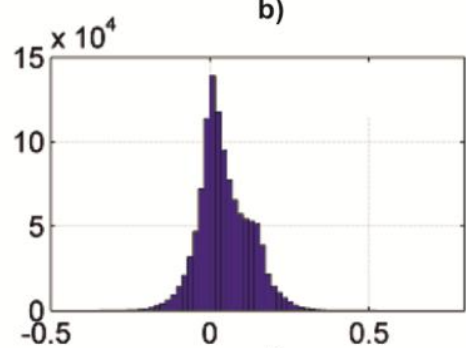

d)

Fig. 2. "High-frequency" coordinate maps (a), (c) and histograms (b), (d) of the linear birefringence distribution inherent to the histological sections of the benign uterine tumor (fibromyoma) biopsy ((a), (b)) and the malignant uterine one (adenocarcinoma) biopsy ((c), (d)).

\subsection{Spatial-frequency Fourier polarimetry of circular} birefringence in the fibrilar networks of uterine tissue

Diagnostic possibilities for differentiation of pathologically changed samples corresponding to the uterine tissue are illustrated with a series in Fig. 2.

Quantitative differences between phase maps of circular birefringence of the endometrium collagenous networks are illustrated by the average values and standard deviations of statistic set $Z_{i=1 ; 2 ; 3 ; 4}$ parameters adduced in Table 2 .
Table 2. Parameters of the statistic, correlative and selfsimilar structure of phase maps of circular birefringence in the polycrystalline uterine tissue networks.

\begin{tabular}{|c|c|c|c|}
\hline \multirow{2}{*}{ Parameters } & \multicolumn{2}{|c|}{$\theta(m \times n)$} & \multirow{2}{*}{$S p, \%$} \\
\cline { 2 - 3 } & Fibromyoma & Adenocarcinoma & \\
\hline$Z_{1}$ & $0.11 \pm 0.021$ & $0.21 \pm 0.037$ & 73 \\
\hline$Z_{2}$ & $0.14 \pm 0.018$ & $0.23 \pm 0.035$ & 81 \\
\hline$Z_{3}$ & $0.79 \pm 0.11$ & $1.38 \pm 0.39$ & 86 \\
\hline$Z_{4}$ & $0.81 \pm 0.13$ & $1.21 \pm 0.21$ & 83 \\
\hline
\end{tabular}


A comparative analysis revealed the following oncologic condition with a high level of specificity $(73 \leq S p \leq 86 \%)$ of uterus parameters (depicted in grey color in Table 2):

- $\quad$ statistic moments of the $1^{\text {st }}$ and $2^{\text {nd }}$ orders $\theta(m \times n)$, differences between values are from 2 up to 3.5 times.

\section{Conclusions}

The model for generalized optic anisotropy in the fibrillar protein matrices has been suggested, and the method of Fourier polarimetry for determining the parameters of linear and circular birefringence in the tissue biopsy taken from uterine wall has been substantiated.

The set of criteria for differentiation of benign (fibromyoma) and malignant (adenocarcinoma) conditions of the uterine has been revealed and substantiated:

statistical moments of the $1^{\text {st }}$ to $4^{\text {th }}$ orders that characterize distribution of the phase shifts between orthogonal components of the laser radiation amplitude, related with linear birefringence of myosin fibres in the endometrium layer;

statistical moments of the $1^{\text {st }}$ to $4^{\text {th }}$ orders that characterize distribution of plane rotations of laser radiation polarization, conditioned by circular birefringence of the network of collagenic fibrils in the endometrium layer.

\section{Acknowledgement}

This work was supported by the grants №0116U001446, №0116U001449, №0115U003241, №0115U003227, №0115U003235 from the Ukraine Foundation for Basic Research.

\section{References}

1. O.V. Angelsky, A.Ya. Bekshaev, P.P. Maksimyak, A.P. Maksimyak, S.G. Hanson, Measurement of small light absorption in microparticles by means of optically induced rotation // Opt. Exp. 23(6), p. 7152-7163 (2015).

O.V. Angelsky, A.Ya. Bekshaev, P.P. Maksimyak, A.P. Maksimyak, S.G. Hanson, C.Yu. Zenkova, Self-action of continuous laser radiation and Pearcey diffraction in a water suspension with light-absorbing particles // Opt. Exp. 22(3), p. 2267-2277 (2014).

3. V.V. Tuchin, Handbook of Coherent-Domain Optical Methods. Biomedical Diagnostics, Environmental and Material Science. Kluwer Academic Publishers, 2004.

4. J.F. de Boer, T.E. Milner, M.J. van Gemert, J.S. Nelson, Two-dimensional birefringence imaging in biological tissue using polarizationsensitive optical coherence tomography // Proc. SPIE, 3196, p. 32-37 (1998).
5. J.F. de Boer, T.E. Milner, M.G. Ducros, S.M. Srinivas and J.S. Nelson, Handbook of Optical Coherence Tomography. Marcel Dekker Inc., New York, 2002, p. 237-274.

6. M.J. Everett, K. Shoenenberger, B.W. Colston, L.B. Da Silva, Birefringence characterization of biological tissue by use of optical coherence tomography // Opt. Lett. 23, p. 228-230 (1998).

7. A.G. Ushenko, Polarization structure of biospeckles and the depolarization of laser radiation // Optics and Spectroscopy, 89(4), p. 597 -600(2000).

8. Yu.O. Ushenko, O.V. Dubolazov A.O. Karachevtsev, M.P. Gorsky, Y.F. Marchuk, Wavelet analysis of Fourier polarized images of the human bile // Appl. Opt. 51(10), p. C133-C139 (2012).

9. Yu.A. Ushenko, V.A. Ushenko, A.V. Dubolazov, V.O. Balanetskaya, N.I. Zabolotna, Mueller-matrix diagnostics of optical properties of polycrystalline networks of human blood plasma // Optics and Spectroscopy, 112(6), p. 884-892 (2012).

10. Yu.A. Ushenko, Yu.Ya. Tomka, A.V. Dubolazov, Laser diagnostics of anisotropy in birefringent networks of biological tissues in different physiological conditions // Quantum Electronics, 41(2), p. 170-175 (2011).

11. Yu.A. Ushenko, A.V. Dubolazov, V.O. Balanetskaya, A.O. Karachevtsev, V.A. Ushenko, Waveletanalysis of polarization maps of human blood plasma // Optics and Spectroscopy, 113(3), p. 332343 (2012).

12. A.G. Ushenko, Laser probing of biological tissues and the polarization selection of their images // Optics and Spectroscopy, 91(6), p. 932-936 (2001).

13. O.V. Angelsky, R.N. Besaha, A.I. Mokhun, I.I. Mokhun, M.O. Sopin, M.S. Soskin, M.V. Vasnetsov // Singularities in vectoral fields // Proc. SPIE, 3904, p. 40 (1999).

14. V.A. Ushenko, M.I. Sidor, Y.F. Marchuk, N.V. Pashkovskaya, D.R. Andreichuk, Azimuthinvariant Mueller-matrix differentiation of the optical anisotropy of biological tissues // Optics and Spectroscopy, 117(1), p. 152-157 (2014).

15. V.A. Ushenko, N.I. Zabolotna, S.V. Pavlov, D.M. Burcovets, O.Yu. Novakovska, Muellermatrices polarization selection of two-dimensional linear and circular birefringence images // Proc. SPIE, 9066, Eleventh International Conference on Correlation Optics, 90661X (2013).

16. V.A. Ushenko, M.P. Gorsky, Complex degree of mutual anisotropy of linear birefringence and optical activity of biological tissues in diagnostics of prostate cancer // Optics and Spectroscopy, 115(2), p. 290-297 (2013).

17. Yu.A. Ushenko, M.P. Gorsky, A.V. Dubolazov, A.V. Motrich, V.A. Ushenko, M.I. Sidor, Spatialfrequency Fourier polarimetry of the complex degree of mutual anisotropy of linear and circular birefringence in the diagnostics of oncological 
changes in morphological structure of biological tissues // Quantum Electronics, 42(8), p. 727 (2012).

18. V.A. Ushenko, Complex degree of mutual coherence of biological liquids, in: ROMOPTO International Conference on Micro- to NanoPhotonics III (pp. 88820V-88820V). International Society for Optics and Photonics, 2013.

19. A.G. Ushenko, Laser diagnostics of biofractals // Quantum Electronics, 29(12), p. 1074-1077 (1999).

20. O.V. Angelsky, Yu.Ya. Tomka, A.G. Ushenko, Y.G. Ushenko, S.B. Yermolenko, 2-D tomography of biotissue images in pre-clinic diagnostics of their pre-cancer states // Proc. SPIE, 5972, p. 158-162 (2005).

21. J. W. Goodman, Statistical properties of laser speckle patters. In: Laser Speckle and Related Phenomena, Ed. J.C. Dainty. Berlin, SpringerVerlag, 1975, p. 9-75.

22. S.C. Cowin, How is a tissue built // J. Biomed. Eng. 122, p. 553-568 (2000).

23. O.V. Angelsky, A.G. Ushenko, Y.G. Ushenko, Complex degree of mutual polarization of biological tissue coherent images for the diagnostics of their physiological state // J. Biomed. Opt. 10(6), p. 060502-060502-3 (2005).

24. O.V. Angelsky, P.V. Polyanskii, S.G. Hanson, Singular optical coloring of regularly scattered white light // Opt. Exp. 14(17), p. 7579-7586 (2006).

25. O.V. Angel'skii, A.G. Ushenko, S.B. Ermolenko, Yu.A. Ushenko, O.V. Pishak, Polarization based visualization of multifractal structures for the diagnostics of pathological changes in biological tissues // Optics and Spectroscopy, 89(5), p. 799804 (2000).

26. A. Gerrard, J.M. Burch, Introduction to Matrix Methods in Optics. New York, A Wiley-Intersci. Publ., 1975.

27. O.V. Angel'skii, A.G. Ushenko, A.D. Arkhelyuk, S.B. Ermolenko, D.N. Burkovets, Scattering of laser radiation by multifractal biological structures // Optics and Spectroscopy, 88(3), p. 444-447 (2000).

28. M. Born, E. Wolf, Principles of Optics. Cambridge Univ. Press, 1999.

29. O.V. Angelsky, P.V. Polyanskii, C.V. Felde, The emerging field of correlation optics // Optics and Photonics News, 23(4), p. 25-29 (2012). 